\title{
Solving Fully Fuzzy Linear System with the Necessary and Sufficient Condition to have a Positive Solution
}

\author{
Ghassan Malkawi*, Nazihah Ahmad and Haslinda Ibrahim \\ College of Arts \& Sciences, University Utara Malaysia, 06010 Sintok, Kedah, Malaysia
}

Received: 11 May. 2013, Revised: 15 Sep. 2013, Accepted: 16 Sep. 2013

Published online: 1 May. 2014

\begin{abstract}
This paper proposes new matrix methods for solving positive solutions for a positive Fully Fuzzy Linear System (FFLS). All coefficients on the right hand side are collected in one block matrix, while the entries on the left hand side are collected in one vector. Therefore, the solution can be gained with a non-fuzzy common step. The necessary theorems are derived to obtain a necessary and sufficient condition in order to obtain the solution. The solution for FFLS is obtained where the entries of coefficients are unknown. The methods and results are also capable of solving Left-Right Fuzzy Linear System (LR-FLS). To best illustrate the proposed methods, numerical examples are solved and compared to the existing methods to show the efficiency of the proposed method. New numerical examples are presented to demonstrate the contributions in this paper.
\end{abstract}

Keywords: Fully Fuzzy Linear System, Sufficient Condition, Positive Solution

\section{Introduction}

System of linear equations has a wide application in varying subjects including mathematics, physics, statistics, operational research, engineering, economics, finance and social sciences. Nevertheless, most of these applications are characterized by the lacking of the imprecision system coefficients and improper information on actual parameters values. Therefore, there is a need to review the mathematical models and numerical variable that are suitable to deal with these ambiguous values; for examples, researchers may introduce fuzzy numbers instead of crisp numbers. Most importantly, it is possible to solve fuzzy linear system (FLS) when the matrix elements are crisp numbers while the right hand side is a fuzzy vector. On the other hand it is called FFLS when both the matrix elements and vector are fuzzy numbers.

The first achievable approach of FLS was obtained by Friedman et al. [14] they proposed a generic model for solving a $n \times n$ FLS by employing the embedding approach. The original FLS was replaced by a $2 n \times 2 n$ crisp linear system and obtained the solution.

Dehghan et al. [12] introduced several methods for solving FFLS that are similar to the classic methods derived from Linear Algebra, such as the LU decomposition and Cramer's rule in order to determine the approximated solution of a system. He also shared a new method of using Linear Programming (LP) in order to obtain the solution of square and non-square matrix. The Adomian decomposition method was also expanded in order to solve the positive fuzzy vector solution of FFLS in [11]. Dehghan and Hashemi in [13] investigated the iterative solution like Gauss-Seidel, Jacobi and Jacobi over-relaxation (JOR).

Few researchers commented on new methods to solve FFLS [21-25], and brought forward new methods to solve FFLS. However, Kumar et al. [4] introduced a new computational method to solve FFLS by relying on the computation of row reduced echelon form. The contribution of their method is that it can easily check the consistency of the system, nique, infinite or no solution.

In this paper, new methods are proposed to solve FFLS through two computational steps. Firstly, we rearrange the coefficients in left hand side to make it easy to transfer from fuzzy system to an associated linear system. Secondly, the problem is solved by the classical methods in Linear Algebra. Unlike the existing methods, the equations are rearranged and the matrices are

\footnotetext{
*Corresponding author e-mail: gassan_malkawi@yahoo.com
} 
represented in a square block matrix. Thereafter, the classical method such as the matrix inverse method is used to find the solution. Through these new methods, we can study the fuzzy solution using the associated linear system, and the conditions for the system to have a consistent solution.

The structure of this paper is organized as follows. In Section 2, the basic definitions of the fuzzy set theory and matrix theory are discussed. This is to explain the concept of FFLS and operations of block matrices. In Section 3, we review the solution of FFLS and introduce the new methods. The theorems and lemmas are derived to obtain a necessary and sufficient condition to prove the existence of solution. Also the solution of LR-FLS is discussed. In Section 4, we conclude the paper.

\section{Preliminaries}

In this section, basic definitions and notions of fuzzy set theory are reviewed [5], [1]:

Definition 2.1. Let $X$ be a universal set. Then, we define the fuzzy subset $\tilde{A}$ of $X$ by its membership function $\mu_{\tilde{A}}: X \rightarrow[0,1]$ which assigns to each element $x \in X a$ real number $\mu_{\tilde{A}}(x)$ in the interval $[0,1]$; where the value $\mu_{\tilde{A}}(x)$ represents the grade of membership of $x$ in $\tilde{A}$. A fuzzy set $\tilde{A}$ is written as:

$$
\tilde{A}=\left\{\left(x, \mu_{\tilde{A}}(x)\right), x \in X, \mu_{\tilde{A}}(x) \in[0,1]\right\} .
$$
set if:

Definition 2.2. A fuzzy set $\tilde{A}$ in $X=\mathbb{R}^{n}$ is convex fuzzy

$$
\begin{gathered}
\forall x_{1}, x_{2} \in X, \forall \lambda \in[0,1], \\
\mu_{\tilde{A}}\left(\lambda x_{1}+(1-\lambda) x_{2}\right) \geq \min \left(\mu_{\tilde{A}}\left(x_{1}\right), \mu_{\tilde{A}}\left(x_{2}\right)\right) .
\end{gathered}
$$

Definition 2.3. Let $\tilde{A}$ be a fuzzy set defined on the set of real numbers $\mathbb{R}$. $\tilde{A}$ is called normal fuzzy set if there exist $x \in \mathbb{R}$ such that $\mu_{\tilde{A}}(x)=1$.

A fuzzy number $\tilde{m}$ is called Left-Right Fuzzy number (LR fuzzy number) where its membership function satisfy

$$
\mu_{\tilde{A}}(x)=\left\{\begin{aligned}
L\left(\frac{a_{1}-x}{\alpha}\right), & x \leq a_{1} \quad \alpha>0, \\
1, & a_{1} \leq x \leq a_{2}, \\
R\left(\frac{x-a_{2}}{\beta}\right), & a_{2} \leq x \quad \beta>0,
\end{aligned}\right.
$$

where $m, \alpha, \beta \in \mathbb{R}$. And the function L(.) is called a left shape function if the following hold

$$
\begin{aligned}
& -L(x)=L(-x), \\
& -L(0)=1, L(1)=0,
\end{aligned}
$$

-L is non-increasing on $[0, \infty]$.

Also the definition of function $R($.$) which called right$ shape is similar to that of $L($.$) . It is symbolically written$ $\tilde{m}=(m, \alpha, \beta)_{L R}$, where $m$ symbolizes the mean value, while $\alpha$ and $\beta$ are left and right spreads, respectively. We denote the set of LR fuzzy numbers $F(R)$.

Definition 2.4. Two fuzzy numbers $\tilde{m}=(m, \alpha, \beta)_{L R}$ and $\tilde{n}=(n, \gamma, \delta)_{L R}$ are called equal, if $n=m, \gamma=\alpha, \delta=$ $\beta$.

Definition 2.5. (Arithmetic operations on LR fuzzy numbers) We will represent arithmetic operations for two LR Fuzzy numbers $\tilde{m}=(m, \alpha, \beta)_{L R}$ and $\tilde{n}=(n, \gamma, \delta)_{L R}$ as follows:

-Addition:

$$
\tilde{m} \oplus \tilde{n}=(m+n, \alpha+\gamma, \beta+\delta)_{L R} .
$$

-Opposite:

$$
-\tilde{m}=-(m, \alpha, \beta)_{L R}=(-m, \beta, \alpha)_{R L} .
$$

-Subtraction:

$$
\tilde{m} \ominus \tilde{n}=(m-n, \alpha+\delta, \beta+\gamma)_{L R} .
$$

\section{-Approximate multiplication:}

$$
\begin{aligned}
& \text { If } \tilde{m}>0 \text { and } \tilde{n}>0 \text { then, } \\
& \quad \tilde{m} \otimes \tilde{n} \cong(m n, m \gamma+n \alpha, m \delta+n \beta)_{L R} .
\end{aligned}
$$

If $\tilde{m}<0$ and $\tilde{n}<0$ then,

$$
\tilde{m} \otimes \tilde{n} \cong(m n,-n \beta-m \delta,-n \alpha-m \gamma)_{R L}
$$

If $\tilde{m}>0$ and $\tilde{n}<0$ then,

$$
\tilde{m} \otimes \tilde{n} \cong(m n, n \alpha-m \delta, n \beta-m \gamma)_{R L}
$$

\section{-Scalar multiplication:}

Let $\lambda \in \mathbb{R}$, then,

$$
\lambda \otimes \tilde{m}=\left\{\begin{array}{c}
(\lambda m, \lambda \alpha, \lambda \beta)_{L R}, \quad \lambda \geq 0, \\
(\lambda m,-\lambda \beta,-\lambda \alpha)_{R L}, \quad \lambda<0 .
\end{array}\right.
$$

Definition 2.6. A popular LR fuzzy number is a triangular fuzzy number (TFN), where

$$
L=R=\max (0,1-x),
$$



by:

consequently, Its membership function in (1) is given

$$
\mu_{\tilde{A}}(x)=\left\{\begin{array}{c}
1-\frac{m-x}{\alpha}, m-\alpha \leq x<m, \alpha>0, \\
1-\frac{x-m}{\beta}, m \leq x<m+\beta, \beta>0, \\
0 \quad \text { otherwise, }
\end{array}\right.
$$

and it is symbolically written as a triangular fuzzy number $\tilde{m}=(m, \alpha, \beta)$.

Definition 2.7. A vector $\tilde{X}=\left(\tilde{x}_{1}, \tilde{x}_{2}, \ldots, \tilde{x}_{n}\right)$, where $\tilde{x}_{i}, 1 \leq i \leq n$ are LR fuzzy numbers, is called an LR fuzzy vector.

Definition 2.8. A matrix $\tilde{A}=\left(\tilde{a}_{i j}\right)_{i, j=1}^{n}$ is called a fuzzy number matrix, or shortly fuzzy matrix, if each element of $\tilde{A}$ is a fuzzy number.

Definition 2.9. Let $\tilde{A}=\left(\tilde{a}_{i j}\right)$ and $\tilde{B}=\left(\tilde{b}_{i j}\right)$ be two $m \times$ $n$ and $n \times p$ respectively. We define $\tilde{A} \otimes \tilde{B}=\tilde{C}=\left(\tilde{c}_{i j}\right)$ which is the $m \times p$ matrix where

$$
\tilde{c}_{i j}=\sum_{k=1, \ldots, n}^{\oplus} \tilde{a}_{i k} \otimes \tilde{b}_{k j}
$$

Definition 2.10. (Fully fuzzy linear system) Consider the $n \times n$ linear system,

$$
\left\{\begin{array}{c}
\tilde{a}_{11} \tilde{x}_{1}+\tilde{a}_{12} \tilde{x}_{2}+\ldots+\tilde{a}_{1 n} \tilde{x}_{n}=\tilde{b}_{1}, \\
\tilde{a}_{21} \tilde{x}_{1}+a_{22} \tilde{x}_{2}+\ldots+\tilde{a}_{2 n} \tilde{x}_{n}=\tilde{b}_{2}, \\
\vdots \\
\vdots \\
\tilde{a}_{n 1} \tilde{x}_{1}+\tilde{a}_{n 2} \tilde{x}_{2}+\ldots+\tilde{a}_{n n} \tilde{x}_{n}=\tilde{b}_{n},
\end{array}\right.
$$

where $\forall \tilde{a}_{i j}, \tilde{b}_{j} \in F(R)$ this system is called a fully fuzzy linear system $(F F L S)$. The matrix $\tilde{A}=\left(\tilde{a}_{i j}\right)_{i, j=1}^{n}$ and the vector $\tilde{B}=\left(\tilde{b}_{j}\right)_{j=1}^{n}$ may be represented as

$$
\tilde{A} \otimes \tilde{X}=\tilde{B}
$$

If the all entries of $\tilde{A}, \tilde{B} \geq 0$ it is called positive FFLS. If the vector $\tilde{X}=\left(\tilde{x}_{j}\right)_{j=1}^{n}$ satisfies (12), and all entries of $\tilde{X}=\left(\tilde{x}_{j}\right)$ are positives, where $\forall \tilde{x}_{j} \in F(R), j=1,2, \ldots, n$, it is called positive fuzzy solution, abbreviated $(P-\tilde{X})$. If $\exists \tilde{x}_{j} \notin F(R), j=1,2, \ldots, n$, it is called non fuzzy solution.

\section{Solving FFLS}

Now we are going to solve FFLS by using the matrix theory. A necessary and sufficient conditions for FFLS to have a positive solution are investigated. At the end of this section LR-FLS is solved using the new method.

\subsection{The proposed method for solving FFLS}

In order to solve (12), we assume that $\tilde{A}, \tilde{X}, \tilde{B} \geq 0$, where $\tilde{A}=(A, M, N), \tilde{X}=(x, y, z)$ and $\tilde{B}=(b, h, g)$, then

$$
(A, M, N) \otimes(x, y, z)=(b, h, g) .
$$

By Arithmetic operations on fuzzy numbers we have,

$$
\left\{\begin{array}{c}
A x=b, \\
A y+M x=h, \\
A z+N x=g .
\end{array}\right.
$$

First, we will find the associated linear system for fuzzy system. The system in (12) can be represented by these equations

$$
\left\{\begin{array}{c}
a_{i 1} x_{1}+a_{i 2} x_{2}+\cdots+a_{i n} x_{n}=b_{i} \\
\left(a_{i 1} y_{1}+a_{i 2} y_{2}+\cdots+a_{i n} y_{n}\right)+ \\
\left(m_{i 1} x_{1}+m_{i 2} x_{2}+\cdots+m_{i n} x_{n}\right)=h_{i} \\
\left(a_{i 1} z_{1}+a_{i 2} z_{2}+\cdots+a_{i n} z_{n}\right)+ \\
\left(n_{i 1} x_{1}+n_{i 2} x_{2}+\cdots+n_{i n} x_{n}\right)=g_{i}
\end{array}\right.
$$

Let us rearrange the linear system and add zero terms as follows:

$$
\left\{\begin{array}{c}
\left(a_{i 1} x_{1}+a_{i 2} x_{2}+\cdots+a_{i n} x_{n}\right)+ \\
(0+0+\cdots+0)+ \\
(0+0+\cdots+0)=b_{i}, \\
\left(m_{i 1} x_{1}+m_{i 2} x_{2}+\cdots+m_{i n} x_{n}\right)+ \\
\left(a_{i 1} y_{1}+a_{i 2} y_{2}+\cdots+a_{i n} y_{n}\right)+ \\
(0+0+\cdots+0)=h_{i}, \\
\left(n_{i 1} x_{1}+n_{i 2} x_{2}+\cdots+n_{i n} x_{n}\right)+ \\
(0+0+\cdots+0)+ \\
\left(a_{i 1} y_{1}+a_{i 2} y_{2}+\cdots+a_{i n} y_{n}\right)=g_{i},
\end{array}\right.
$$

where $1 \leq i \leq n$.

Appoint the block $S=\left(s_{i, j}\right)_{3 n \times 3 n}$ matrix where $1 \leq$ $i \leq n$ as follows:

$$
\left\{\begin{array}{c}
s_{i, j}=s_{i+n, j+n}=s_{i+2 n, j+2 n}=a_{i j} \\
s_{i+n, j}=m_{i j} \\
s_{i+2 n, j}=n_{i j}
\end{array}\right.
$$

Also, any $s_{i j}$ which is undetermined in (17) is zero. Then we have a new $3 n \times 3 n$ block matrix $S$ : 


$$
S=\left(\begin{array}{ccc}
A & 0 & 0 \\
M & A & 0 \\
N & 0 & A
\end{array}\right)
$$

Definition 3.1. (The associated linear system) Let the block matrix $S$ be as follows,

$$
S=\left(\begin{array}{lll}
A & 0 & 0 \\
M & A & 0 \\
N & 0 & A
\end{array}\right)
$$

where $A, M$ and $N$ square matrices are in common size n. Also let

$$
X=\operatorname{Vec}(x, y, z)=\left(\begin{array}{l}
x \\
y \\
z
\end{array}\right)
$$

and,

$$
B=\operatorname{Vec}(b, h, g)=\left(\begin{array}{l}
b \\
h \\
g
\end{array}\right),
$$

where $x, y, z, b, h$ and $g$ are vectors of $n$ components. We will appoint new $3 n \times 3 n$ linear system,

$$
S X=B,
$$

in matrix form,

$$
\left(\begin{array}{ccc}
A & 0 & 0 \\
M & A & 0 \\
N & 0 & A
\end{array}\right) \operatorname{Vec}(x, y, z)=\operatorname{Vec}(b, h, g),
$$

hence, the new linear system can be written by:

$$
\left(\begin{array}{lll}
A & 0 & 0 \\
M & A & 0 \\
N & 0 & A
\end{array}\right)\left(\begin{array}{l}
x \\
y \\
z
\end{array}\right)=\left(\begin{array}{l}
b \\
h \\
g
\end{array}\right)
$$

where,

$$
\begin{aligned}
& A=\left(\begin{array}{ccc}
a_{11} & \ldots & a_{1 n} \\
\vdots & \ddots & \vdots \\
a_{n 1} & \cdots & a_{n n}
\end{array}\right) \\
& M=\left(\begin{array}{ccc}
m_{11} & \ldots & m_{1 n} \\
\vdots & \ddots & \vdots \\
m_{n 1} & \cdots & m_{n n}
\end{array}\right), \\
& N=\left(\begin{array}{ccc}
n_{11} & \ldots & n_{1 n} \\
\vdots & \ddots & \vdots \\
n_{n 1} & \cdots & n_{n n}
\end{array}\right) \\
& x=\left(\begin{array}{c}
x_{1} \\
\vdots \\
x_{n}
\end{array}\right), y=\left(\begin{array}{c}
y_{1} \\
\vdots \\
y_{n}
\end{array}\right), z=\left(\begin{array}{c}
z_{1} \\
\vdots \\
z_{n}
\end{array}\right) \text {, } \\
& b=\left(\begin{array}{c}
b_{1} \\
\vdots \\
b_{n}
\end{array}\right), h=\left(\begin{array}{c}
h_{1} \\
\vdots \\
h_{n}
\end{array}\right), g=\left(\begin{array}{c}
g_{1} \\
\vdots \\
g_{n}
\end{array}\right) \text {. }
\end{aligned}
$$

In this paper, the linear system $S X=B$, is called the associated linear system for FFLS.

In order to prove few theorems in this section we have to review the operation block matrices, since the techniques used for manipulating block matrices are similar for ordinary matrices. As an application, the elementary row or column operations for ordinary matrices can be generalized to block or partitioned matrices as follows [6]:

1.By interchanging two block rows or columns.

2.By multiplying a block row or columns from the left or right by a non-singular matrix of appropriate size.

3.By multiplying a block row or column by a non-zero matrix from the left or right, then add it to another row or column.

As such, there is a necessity to show the relation between the solution for the associated crisp system in Definition 3.1. and FFLS. Therefore the following two statements based on the techniques for block matrices reviewed:

-The $3 n \times 3 n$ crisp linear system in (18) can be uniquely obtained the solution $X$, if and only if the matrix $S$ is nonsingular.

-Are the components of $3 n$ dimensional crisp solution vector $X$ in (20) representing a corresponding solution $\tilde{X}$ of fuzzy system? In other words, is the solution for $S X=B$ also a solution for FFLS.

The next theorem determines when crisp matrix $S$ is nonsingular.

Theorem 3.1. The block matrix $S$ in (18) is non-singular if and only if the matrix $A$ in (13) is non-singular.

\section{Proof}

Since $A, M$ and $N$ are square matrices in common order $n$, we can easily make the matrix $S$ a diagonal block matrix by subtracting the first row multiplied by $M A^{-1}$ from the second row, and subtracting the first row multiplied by $N A^{-1}$ from the third row as follows:

$$
S=\left(\begin{array}{ccc}
A & 0 & 0 \\
M & A & 0 \\
N & 0 & A
\end{array}\right) \rightarrow\left(\begin{array}{ccc}
A & 0 & 0 \\
0 & A & 0 \\
0 & 0 & A
\end{array}\right)=S_{1}
$$

Now, we will expand $S_{1}$ through 3 block matrices of order $3 n \times 3 n,\left(E_{1}, E_{2}, E_{3}\right)$ where,

$$
\begin{aligned}
& E_{1}=\left(\begin{array}{ccc}
A & 0 & 0 \\
0 & I_{n} & 0 \\
0 & 0 & I_{n}
\end{array}\right), \\
& E_{2}=\left(\begin{array}{ccc}
I_{n} & 0 & 0 \\
0 & A & 0 \\
0 & 0 & I_{n}
\end{array}\right),
\end{aligned}
$$




$$
E_{3}=\left(\begin{array}{ccc}
I_{n} & 0 & 0 \\
0 & I_{n} & 0 \\
0 & 0 & A
\end{array}\right)
$$

Hence,

$$
S_{1}=E_{1} E_{2} E_{3}
$$

Clearly,

$$
|S|=\left|S_{1}\right|=\left|E_{1}\right|\left|E_{2}\right|\left|E_{3}\right|=|A|^{3} .
$$

Therefore,

$$
|S| \neq 0 \text { if and only if }|A| \neq 0 .
$$

Moreover,

$$
|S|=|A|^{3}
$$

And this concludes the proof.

Now we will study the equivalency between the fuzzy system and the associated crisp linear system.

Theorem 3.2. The unique solutions of $S X=B$ in (18) and $\tilde{A} \otimes X=\tilde{B}$ in (12) are equivalent.

Proof Let $A$ be an invertible matrix in (13). A row reduced echelon form of augmented $(G \mid B)$ is employed to compute the solution.

$$
\begin{aligned}
& S=\left(\begin{array}{ccccc}
A & 0 & 0 & \vdots & b \\
M & A & 0 & \vdots & h \\
N & 0 & A & & g
\end{array}\right) \rightarrow \\
& \left(\begin{array}{ccccc}
I & 0 & 0 & \vdots & A^{-1} b \\
M & A & 0 & \vdots & h \\
N & 0 & A & & \\
& & & \vdots &
\end{array}\right)=S_{1}
\end{aligned}
$$

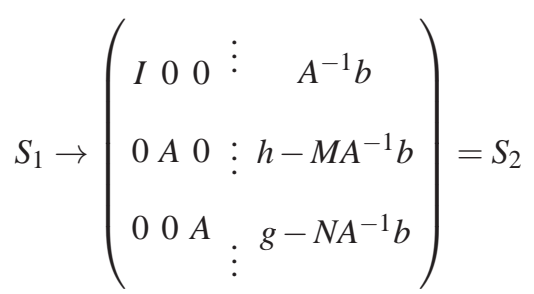

$$
\begin{aligned}
& S_{2} \rightarrow\left(\begin{array}{cccccc}
I & 0 & 0 & \vdots & A^{-1} b \\
0 & I & 0 & \vdots & A^{-1}\left(h-M A^{-1} b\right) \\
& & 0 & I & & \\
0 & & & \vdots & A^{-1} & \left(g-N A^{-1} b\right)
\end{array}\right)=S_{3},
\end{aligned}
$$

the solution of $S X=B$ is

$$
=\left(\begin{array}{c}
A^{-1} b \\
A^{-1}\left(h-M A^{-1} b\right) \\
A^{-1}\left(g-N A^{-1} b\right)
\end{array}\right),
$$

then,

$$
\left(\begin{array}{c}
A^{-1} b \\
A^{-1} h-A^{-1} M A^{-1} b \\
A^{-1} g-A^{-1} N A^{-1} b
\end{array}\right)=\left(\begin{array}{l}
x \\
y \\
z
\end{array}\right) .
$$

Now, from (14) we can get $x, y$ and $z$ by multiplying each equation by $A^{-1}$,

$$
\begin{gathered}
x=A^{-1} b, \\
y+A^{-1} M x=A^{-1} h, \\
z+A^{-1} N x=A^{-1} g .
\end{gathered}
$$

By substituting $x=A^{-1} b$ in second and third equations

$$
\begin{gathered}
y+A^{-1} M A^{-1} b=A^{-1} h, \\
z+A^{-1} N A^{-1} b=A^{-1} g .
\end{gathered}
$$

Hence after rearranging the equations we get

$$
\begin{aligned}
& y=A^{-1} h-A^{-1} M A^{-1} b, \\
& z=A^{-1} g-A^{-1} N A^{-1} b .
\end{aligned}
$$

Then $x, y$ and $z$ are equivalent to the proposed solution of $P-X$ for $P-F F L S$ in (22).

Corollary 3.1. If the associated linear system does not have a unique solution then the FFLS does not have one either.

The following examples show that the solution can be obtained directly form (20).

Example 3.1. Find a positive solution for the following FFLS, [12].

$(6,1,4) \otimes\left(x_{1}, y_{1}, z_{1}\right) \oplus(5,2,2) \otimes\left(x_{2}, y_{2}, z_{2}\right) \oplus$ $(3,2,1) \otimes\left(x_{3}, y_{3}, z_{3}\right)=(58,30,60)$,

$(12, \quad 8, \quad 20) \otimes\left(x_{1}, \quad y_{1}, \quad z_{1}\right) \oplus(14, \quad 12, \quad 15) \otimes$ $\left(x_{2}, \quad y_{2}, \quad z_{2}\right) \oplus(8, \quad 8, \quad 10) \otimes\left(x_{3}, \quad y_{3}, \quad z_{3}\right)=$ $(142, \quad 139, \quad 257), \quad(24, \quad 10, \quad 34) \otimes\left(x_{1}, \quad y_{1}, \quad z_{1}\right) \oplus$ $(32, \quad 30, \quad 30) \otimes\left(x_{2}, \quad y_{2}, \quad z_{2}\right) \oplus(20, \quad 19, \quad 24) \otimes$ $\left(x_{3}, y_{3}, z_{3}\right)=(316,297,514)$.

Where $\tilde{x}_{i}=\left(x_{i}, y_{i}, z_{i}\right) \geq 0, i=1,2,3$.

Solution

The system may be written in matrix form, 


$$
\begin{aligned}
& \left(\begin{array}{ccc}
(6,1,4) & (5,2,2) & (3,2,1) \\
(12,8,20) & (14,12,15) & (8,8,10) \\
(24,10,34) & (32,30,30) & (20,19,24)
\end{array}\right) \\
& \left(\begin{array}{l}
\left(x_{1}, y_{1}, z_{1}\right) \\
\left(x_{2}, y_{2}, z_{2}\right) \\
\left(x_{3}, y_{3}, z_{3}\right)
\end{array}\right)=\left(\begin{array}{c}
(58,30,60) \\
(142,139,257) \\
(316,297,514)
\end{array}\right) .
\end{aligned}
$$

where,

$$
\begin{gathered}
A=\left(\begin{array}{ccc}
6 & 5 & 3 \\
12 & 14 & 8 \\
24 & 32 & 20
\end{array}\right), \\
M=\left(\begin{array}{ccc}
1 & 2 & 2 \\
8 & 12 & 8 \\
10 & 30 & 19
\end{array}\right), N=\left(\begin{array}{ccc}
4 & 2 & 1 \\
20 & 15 & 10 \\
34 & 30 & 24
\end{array}\right) . \\
b=\left(\begin{array}{c}
58 \\
142 \\
316
\end{array}\right), h=\left(\begin{array}{c}
30 \\
139 \\
297
\end{array}\right), g=\left(\begin{array}{c}
60 \\
257 \\
514
\end{array}\right), \\
x=\left(\begin{array}{l}
x_{1} \\
x_{2} \\
x_{3}
\end{array}\right), y=\left(\begin{array}{l}
y_{1} \\
y_{2} \\
y_{3}
\end{array}\right), z=\left(\begin{array}{c}
z_{1} \\
z_{2} \\
z_{3}
\end{array}\right) .
\end{gathered}
$$

The associated linear system can be constructed by $S X=B$, where $S, X$ and $B$ are appointed as follows:

$$
S=\left(\begin{array}{ccc}
A & 0 & 0 \\
M & A & 0 \\
N & 0 & A
\end{array}\right),
$$

also,

$$
\left.\begin{array}{rl}
X=\operatorname{Vec}(x, y, z)=\left(\begin{array}{l}
x \\
y \\
z
\end{array}\right)=\left(\begin{array}{l}
\left(\begin{array}{l}
x_{1} \\
x_{2} \\
x_{3}
\end{array}\right) \\
\left(\begin{array}{l}
y_{1} \\
y_{2} \\
y_{3}
\end{array}\right) \\
\left(\begin{array}{c}
z_{1} \\
z_{2} \\
z_{3}
\end{array}\right)
\end{array}\right), \\
B=\operatorname{Vec}(b, h, g)=\left(\begin{array}{l}
5 \\
h \\
g
\end{array}\right)=\left(\begin{array}{c}
142 \\
316
\end{array}\right) \\
\left(\begin{array}{c}
30 \\
139 \\
297
\end{array}\right) \\
\left(\begin{array}{c}
60 \\
257 \\
514
\end{array}\right)
\end{array}\right) .
$$

Since $|A| \neq 0$, according to Theorem 3.1., $|S| \neq 0$.

Then the associated linear system is given by $S X=B$ :

$$
\begin{gathered}
\left(\begin{array}{ccccccccc}
6 & 5 & 3 & 0 & 0 & 0 & 0 & 0 & 0 \\
12 & 14 & 8 & 0 & 0 & 0 & 0 & 0 & 0 \\
24 & 32 & 20 & 0 & 0 & 0 & 0 & 0 & 0 \\
1 & 2 & 2 & 6 & 5 & 3 & 0 & 0 & 0 \\
8 & 12 & 8 & 12 & 14 & 8 & 0 & 0 & 0 \\
10 & 30 & 19 & 24 & 32 & 20 & 0 & 0 & 0 \\
4 & 2 & 1 & 0 & 0 & 0 & 6 & 5 & 3 \\
20 & 15 & 10 & 0 & 0 & 0 & 12 & 14 & 8 \\
34 & 30 & 24 & 0 & 0 & 0 & 24 & 32 & 20
\end{array}\right)\left(\begin{array}{l}
x_{1} \\
x_{2} \\
x_{3} \\
y_{1} \\
y_{2} \\
y_{3} \\
z_{1} \\
z_{2} \\
z_{3}
\end{array}\right) \\
\\
=\left(\begin{array}{c}
58 \\
142 \\
316 \\
30 \\
139 \\
297 \\
60 \\
257 \\
514
\end{array}\right)
\end{gathered}
$$

the crisp solution can be obtained by only computing $X=$ $S^{-1} B$ :

$$
\begin{aligned}
& X=\left(\begin{array}{l}
x_{1} \\
x_{2} \\
x_{3} \\
y_{1} \\
y_{2} \\
y_{3} \\
z_{1} \\
z_{2} \\
z_{3}
\end{array}\right)=\left(\begin{array}{c}
4 \\
5 \\
3 \\
1 \\
\frac{1}{2} \\
\frac{1}{2} \\
2 \\
1
\end{array}\right), \text { or } \\
& X=\left(\begin{array}{l}
\left(\begin{array}{l}
x_{1} \\
x_{2} \\
x_{3}
\end{array}\right) \\
\left(\begin{array}{l}
y_{1} \\
y_{2} \\
y_{3}
\end{array}\right) \\
\left(\begin{array}{l}
z_{1} \\
z_{2} \\
z_{3}
\end{array}\right)
\end{array}\right)=\left(\begin{array}{c}
\left(\begin{array}{l}
4 \\
5 \\
3
\end{array}\right) \\
\left(\begin{array}{c}
1 \\
0.5 \\
0.5
\end{array}\right) \\
\left(\begin{array}{l}
3 \\
2 \\
1
\end{array}\right)
\end{array}\right) .
\end{aligned}
$$

Then the fuzzy solution is 


$$
\begin{aligned}
\tilde{X}= & \left(\begin{array}{l}
\tilde{x}_{1} \\
\tilde{x}_{2} \\
\tilde{x}_{3}
\end{array}\right)=\left(\begin{array}{l}
\left(x_{1}, y_{1}, z_{1}\right) \\
\left(x_{2}, y_{2}, z_{2}\right) \\
\left(x_{3}, y_{3}, z_{3}\right)
\end{array}\right) \\
& =\left(\begin{array}{c}
(4,1,3) \\
(5,0.5,2) \\
(3,0.5,1)
\end{array}\right),
\end{aligned}
$$
3.2 .

where $\tilde{X}$ is a unique solution according to Theorem

Now, the third question to be answered.

-Does the exact solution $\tilde{X}$ satisfying (12) is a positive fuzzy solution for any FFLS?

Before answering the above question, two examples are illustrated here for non-fuzzy solution and non-positive fuzzy solution.

Example 3.2. Find a positive solution for the following FFLS.

$(5,0,1) \otimes\left(x_{1}, y_{1}, z_{1}\right) \oplus(6,1,2) \otimes\left(x_{2}, y_{2}, z_{2}\right)$ $=(50,10,17)$,

$(7,1,0) \otimes\left(x_{1}, y_{1}, z_{1}\right) \oplus(4,0,1) \otimes\left(x_{2}, y_{2}, z_{2}\right)$ $=(48,5,7)$.

Where $\tilde{x}_{i}=\left(x_{i}, y_{i}, z_{i}\right) \geq 0, i=1,2$.

\section{Solution}

The system may be written in matrix form,

$$
\begin{gathered}
\left(\begin{array}{c}
(5,0,1)(6,1,2) \\
(7,1,0)(4,0,1)
\end{array}\right)\left(\begin{array}{l}
\left(x_{1}, y_{1}, z_{1}\right) \\
\left(x_{2}, y_{2}, z_{2}\right)
\end{array}\right) \\
=\left(\begin{array}{c}
(50,10,17) \\
(48,5,7)
\end{array}\right) .
\end{gathered}
$$

Then,

$$
\begin{gathered}
A=\left(\begin{array}{ll}
5 & 6 \\
7 & 4
\end{array}\right), M=\left(\begin{array}{ll}
0 & 1 \\
1 & 0
\end{array}\right), N=\left(\begin{array}{ll}
1 & 2 \\
0 & 1
\end{array}\right), \\
b=\left(\begin{array}{l}
50 \\
48
\end{array}\right), h=\left(\begin{array}{c}
10 \\
5
\end{array}\right), g=\left(\begin{array}{c}
17 \\
7
\end{array}\right),
\end{gathered}
$$

and,

$$
x=\left(\begin{array}{l}
x_{1} \\
x_{2}
\end{array}\right), y=\left(\begin{array}{l}
x_{1} \\
x_{2}
\end{array}\right), z=\left(\begin{array}{l}
z_{1} \\
z_{2}
\end{array}\right) .
$$

Hence, the associated linear system is given by $S X=B$

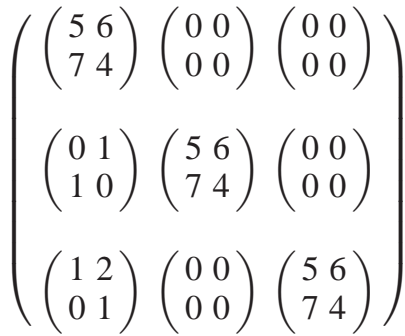

$$
\begin{aligned}
& \left(\begin{array}{l}
\left(\begin{array}{l}
x_{1} \\
x_{2}
\end{array}\right) \\
\left(\begin{array}{l}
y_{1} \\
y_{2}
\end{array}\right) \\
\left(\begin{array}{l}
z_{1} \\
z_{2}
\end{array}\right)
\end{array}\right)=\left(\begin{array}{c}
\left(\begin{array}{c}
50 \\
48
\end{array}\right) \\
\left(\begin{array}{c}
10 \\
5
\end{array}\right) \\
\left(\begin{array}{c}
17 \\
7
\end{array}\right)
\end{array}\right) .
\end{aligned}
$$

By computing $S^{-1} B$ then,

$$
X=\left(\begin{array}{l}
\left(\begin{array}{l}
x_{1} \\
x_{2}
\end{array}\right) \\
\left(\begin{array}{l}
y_{1} \\
y_{2}
\end{array}\right) \\
\left(\begin{array}{l}
z_{1} \\
z_{2}
\end{array}\right)
\end{array}\right)=\left(\begin{array}{c}
4 \\
5
\end{array}\right)\left(\begin{array}{c}
-\frac{7}{11} \\
\frac{15}{11}
\end{array}\right) .
$$

Hence, the unique solution of the fuzzy system is

$$
\begin{gathered}
\tilde{x}_{1}=\left(x_{1}, y_{1}, z_{1}\right)=\left(4,-\frac{7}{11}, 0\right), \\
\tilde{x}_{2}=\left(x_{2}, y_{2}, z_{2}\right)=\left(5, \frac{15}{11}, \frac{1}{2}\right) .
\end{gathered}
$$

Since $y_{1}=-\frac{7}{11}$ is non-positive, the solution is a non-fuzzy solution even though $|S|,|A| \neq 0$. However the vector solution $X$ is unique, but $\tilde{X}$ is still is a non-fuzzy vector.

Example 3.3. Find a positive solution for the following FFLS.

$$
\begin{gathered}
(3.1, \quad 0.4,0.1) \otimes\left(x_{1}, y_{1}, z_{1}\right) \oplus(2,0.6,0.4) \otimes \\
\left(x_{2}, y_{2}, z_{2}\right)=(17.3,14.75,7.86),
\end{gathered}
$$

$(8,0.6,0.7) \otimes\left(x_{1}, y_{1}, z_{1}\right) \oplus(6.9,0.2,0.1) \otimes$

$$
\left(x_{2}, y_{2}, z_{2}\right)=(51.6,32.91,22.72) \text {. }
$$

Where $\tilde{x}_{i}=\left(x_{i}, y_{i}, z_{i}\right) \geq 0, i=1,2$.

\section{Solution}

The system may be written in matrix form, 


$$
\begin{aligned}
& \left(\begin{array}{cc}
(3.1,0.4,0.1) & (2,0.6,0.4) \\
(8,0.6,0.7) & (6.9,0.2,0.1)
\end{array}\right) \\
& \left(\begin{array}{l}
\left(x_{1}, y_{1}, z_{1}\right) \\
\left(x_{2}, y_{2}, z_{2}\right)
\end{array}\right)=\left(\begin{array}{c}
(17.3,14.75,7.86) \\
(51.6,32.91,22.72)
\end{array}\right) .
\end{aligned}
$$

Then,

$$
\begin{gathered}
A=\left(\begin{array}{cc}
3.1 & 2 \\
8 & 6.9
\end{array}\right), \\
M=\left(\begin{array}{ll}
0.4 & 0.6 \\
0.6 & 0.2
\end{array}\right), N=\left(\begin{array}{ll}
0.1 & 0.4 \\
0.7 & 0.1
\end{array}\right), \\
b=\left(\begin{array}{l}
17.3 \\
51.6
\end{array}\right), h=\left(\begin{array}{l}
14.75 \\
32.91
\end{array}\right), g=\left(\begin{array}{c}
7.86 \\
22.72
\end{array}\right),
\end{gathered}
$$

and,

$$
x=\left(\begin{array}{l}
x_{1} \\
x_{2}
\end{array}\right), y=\left(\begin{array}{l}
x_{1} \\
x_{2}
\end{array}\right), z=\left(\begin{array}{l}
z_{1} \\
z_{2}
\end{array}\right) .
$$

Hence, the matrix $S$ is appointed as follows,

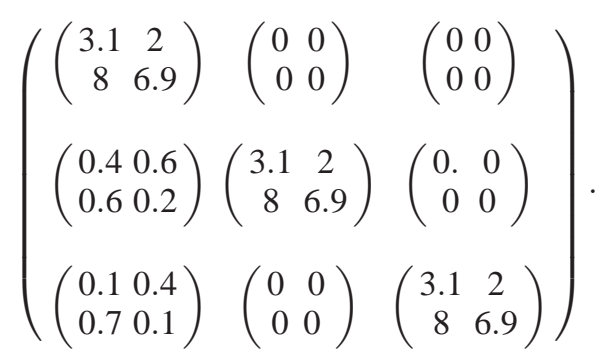

The vector $B$ is appointed as follows,

$$
B=\left(\begin{array}{c}
\left(\begin{array}{l}
17.3 \\
51.6
\end{array}\right) \\
\left(\begin{array}{l}
14.75 \\
32.91
\end{array}\right) \\
\left(\begin{array}{c}
7.86 \\
22.72
\end{array}\right)
\end{array}\right)
$$

The crisp solution of system $X=S^{-1} B$ is

$$
X=\left(\begin{array}{l}
\left(\begin{array}{l}
x_{1} \\
x_{2}
\end{array}\right) \\
\left(\begin{array}{l}
y_{1} \\
y_{2}
\end{array}\right) \\
\left(\begin{array}{l}
z_{1} \\
z_{2}
\end{array}\right)
\end{array}\right)=\left(\begin{array}{c}
\left(\begin{array}{l}
3 \\
4
\end{array}\right) \\
\left(\begin{array}{c}
3.0269 \\
0.883302
\end{array}\right) \\
\left(\begin{array}{c}
0.126902 \\
2.7833
\end{array}\right)
\end{array}\right) .
$$

Then the fuzzy solution is

$$
\begin{aligned}
& \tilde{x}_{1}=\left(x_{1}, y_{1}, z_{1}\right)=(3,3.0269,0.126902), \\
& \tilde{x}_{2}=\left(x_{2}, y_{2}, z_{2}\right)=(4,0.883302,2.7833) .
\end{aligned}
$$

Since $x_{1}-y_{1} \ngtr 0$, then $\left(x_{1}, y_{1}, z_{1}\right)$ is a non-positive LR fuzzy number.

Therefore, we have to determine, the conditions for known positive matrix $\tilde{A}$ and positive vector $\tilde{B}$ to have a positive solution. In the following section, the conditions for FFLS to have positive solution are discussed.

\subsection{A necessary and sufficient conditions for FFLS to have positive solution}

A necessary and sufficient conditions for FFLS to have a positive solution are investigated in the two following cases:

Case 1: The conditions on both $\tilde{A}$ and $\tilde{B}$.

(Dehghan et al. [12]) studied this case using the following theorem.

Theorem 3.3. (Dehghan et al. [12]) let $\tilde{A}=(A, M, N), \tilde{B}=(b, h, g) \geq 0$, and

i- Centric matrix $A$ be an inverse-nonnegative.

i.e. $A^{-1}$ exist and $A^{-1}>0$.

$i i-h \geq M A^{-1} b, g \geq N A^{-1} b$ and

$\left(M \bar{A}^{-1}+I\right) b \geq h$.

Then the system has $P-X$.

Literature review revealed that no investigation, was carried out whether the $P-F F L S$ have $P-X$ or not, even though it is mentioned in most of studies for $(P-F F L S)$. Although [12] illustrated an example of non-fuzzy solution, they couldn't apply their theorem.

Therefore, we can summarize the weakness of this theorem in two points:

1. The further condition $\left(M A^{-1}+I\right) b \geq h$ is used to proof $x-y \geq 0$ in $X=(x, y, z)$.

The following alternative proof shows that $\left(M A^{-1}+I\right) b \geq$ $h$ is redundant, and must be omitted.

$$
\begin{gathered}
b \geq 0 \text { and } A^{-1}>0 \Rightarrow X=A^{-1} b \geq 0, \\
x-y=\left(A^{-1} b\right)-\left(A^{-1} h-A^{-1} M A^{-1} b\right) \\
=A^{-1} b-A^{-1} h+A^{-1} M A^{-1} b .
\end{gathered}
$$

Hence: 


$$
\begin{aligned}
x-y & =A^{-1}\left[(b-h)+\left(M A^{-1} b\right)\right] \\
& =A^{-1}[(b-h)+(M x)],
\end{aligned}
$$

$(b-h) \geq 0$ since $\tilde{b}=(b, h, g)$ is non-negative fuzzy number vector and $A^{-1}, M x \geq 0$,

$$
\text { then } x-y \geq 0 \text {. }
$$

However $\left(M A^{-1}+I\right) b \geq h$ is already satisfied by the hypothesis $A^{-1}>0$,

$$
\begin{gathered}
\left(M A^{-1}+I\right) b-h=\left(M A^{-1} b+b\right)-h \\
=\left(M A^{-1} b\right)+(b-h) \\
\text { since } A^{-1}>0, M, b \geq 0 \text { and }(b-h) \geq 0, \\
\text { then }\left(M A^{-1}+I\right) b-h \geq 0 .
\end{gathered}
$$

2. The condition $A^{-1}>0$ is very powerful, as is shown in the following theorem.

Theorem 3.4. (Minc, [32]) The inverse of a nonnegative matrix $A$ is a nonnegative if and only if $A$ is a generalized permutation matrix.

Therefore, the restriction $A^{-1}>0$ discussed in our case $(P-F F L S)$ by the following result.

Corollary 3.2. If the matrix $A$ in (13) is a generalized permutation matrix then the right spread matrix $M$ is also a generalized permutation matrix, and must have the same structure of A, i.e. $\forall \tilde{a}_{i j}=\left(a_{i j}, m_{i j}, n_{i j}\right)$,

$$
\text { when } a_{i j}=0 \text {, then } m_{i j}=0, \forall i, j=1, \ldots, n \text {. }
$$

\section{Proof}

The proof is obtained easily by contradiction.

Let $\exists m_{i j} \neq 0$, and $a_{i j}=0$. Then,

$a_{i j}-m_{i j} \ngtr 0$. This led to $\tilde{a}_{i j}$ as a negative LR fuzzy number, which contradict with the hypothesis $\forall \tilde{a}_{i j} \geq 0$ in $P-F F L S$. And this concludes the proof.

According to Theorem 3.4. and Corollary 3.2. $A^{-1}>0$ is only satisfied in our case of $(P-F F L S)$ when:

1. The entries of the matrices $A=\left(a_{i j}\right)_{i, j=1}^{n}$ and $M=$ $\left(m_{i j}\right)_{i, j=1}^{n}$ are all zero except for a single positive entry in each row and column.

2. The right spread $m_{i j} \neq 0$ only when $a_{i j} \neq 0$.

In order to point out the weakness of the previous theorem, according to the discussion above, we can illustrate an example in following form of $a_{i j}$ to satisfy Theorem 3.3.

$$
\tilde{a}_{i j}=\left\{\begin{array}{c}
\left(0,0, n_{i j}\right), \text { where } a_{i j}=0 . \\
\left(a_{i j}, m_{i j}, n_{i j}\right), \text { where } a_{i j} \neq 0 .
\end{array}\right.
$$

Example 3.4. Find the positive solution for the following FFLS.

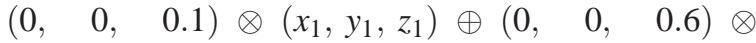
$\left(x_{2}, y_{2}, z_{2}\right) \oplus(0,0,0.2) \otimes\left(x_{3}, y_{3}, z_{3}\right) \oplus(4,2,0.2) \otimes$ $\left(x_{4}, y_{4}, z_{4}\right)=(16,8.4,14.9)$,

$(11,0,0) \otimes\left(x_{1}, y_{1}, z_{1}\right) \oplus(0,0,0.2) \otimes\left(x_{2}, y_{2}, z_{2}\right) \oplus$ $(0,0,0.11) \otimes\left(x_{3}, y_{3}, z_{3}\right) \oplus(0,0,0.13) \otimes\left(x_{4}, y_{4}, z_{4}\right)=$ $(55,7.2,42.07)$,

$(0,0,0.5) \otimes\left(x_{1}, y_{1}, z_{1}\right) \oplus(5,3,0.6) \otimes\left(x_{2}, y_{2}, z_{2}\right) \oplus$ $(0,0,0.9) \otimes\left(x_{3}, y_{3}, z_{3}\right) \oplus(0,0,0.17) \otimes\left(x_{4}, y_{4}, z_{4}\right)=$ $(95,60.5,19.83)$,

$(0,0,0.9) \otimes\left(x_{1}, y_{1}, z_{1}\right) \oplus(0,0,0.9) \otimes\left(x_{2}, y_{2}, z_{2}\right) \oplus$ $(2,0.5,0.7) \otimes\left(x_{3}, y_{3}, z_{3}\right) \oplus(0,0,0.3) \otimes\left(x_{4}, y_{4}, z_{4}\right)=$ $(10,4.3,26.5)$.

Where $\tilde{x}_{i}=\left(x_{i}, y_{i}, z_{i}\right) \geq 0, i=1, \ldots, 4$.

\section{Solution}

The system may be written in matrix form,

$$
\left(\begin{array}{ccc}
A & 0 & 0 \\
M & A & 0 \\
N & 0 & A
\end{array}\right)\left(\begin{array}{l}
x \\
y \\
z
\end{array}\right)=\left(\begin{array}{l}
b \\
h \\
g
\end{array}\right),
$$

where,

$$
\begin{gathered}
A=\left(\begin{array}{cccc}
0 & 0 & 0 & 4 \\
1 & 0 & 0 & 0 \\
0 & 5 & 0 & 0 \\
0 & 0 & 2 & 0
\end{array}\right), M=\left(\begin{array}{llll}
0 & 0 & 0 & 2 \\
0 & 0 & 0 & 0 \\
0 & 3 & 0 & 0 \\
0 & 0 & 0.5 & 0
\end{array}\right), \\
N=\left(\begin{array}{ccccc}
0.1 & 0.6 & 0.2 & 0.2 \\
0 & 0.2 & 0.11 & 0.13 \\
0.5 & 0.6 & 0.9 & 0.17 \\
0.9 & 0.9 & 0.7 & 0.3
\end{array}\right) \\
A^{-1}=\left(\begin{array}{cccc}
0 & \frac{1}{11} & 0 & 0 \\
0 & 0 & \frac{1}{5} & 0 \\
0 & 0 & 0 & \frac{1}{2} \\
\frac{1}{4} & 0 & 0 & 0
\end{array}\right)
\end{gathered}
$$

Also,

$$
\begin{gathered}
b=\left(\begin{array}{l}
16 \\
55 \\
95 \\
10
\end{array}\right), h=\left(\begin{array}{c}
8.4 \\
7.2 \\
60.5 \\
4.3
\end{array}\right), g=\left(\begin{array}{c}
14.9 \\
42.07 \\
19.83 \\
26.5
\end{array}\right), \\
x=\left(\begin{array}{l}
x_{1} \\
x_{2} \\
x_{3} \\
x_{4}
\end{array}\right), y=\left(\begin{array}{l}
y_{1} \\
y_{2} \\
y_{3} \\
y_{4}
\end{array}\right), z=\left(\begin{array}{l}
z_{1} \\
z_{2} \\
z_{3} \\
z_{4}
\end{array}\right) .
\end{gathered}
$$


Since $A^{-1}>0$, we can apply Theorem 3.3.

$$
\begin{gathered}
h-M A^{-1} b=\left(\begin{array}{c}
0.4 \\
7.2 \\
3.5 \\
1.8
\end{array}\right) \geq 0, \\
g-N A^{-1} b=\left(\begin{array}{c}
1.2 \\
37.2 \\
0.75 \\
0.2
\end{array}\right) \geq 0, \\
\left(M A^{-1}+I\right) b-h=\left(\begin{array}{c}
15.6 \\
47.8 \\
91.5 \\
8.2
\end{array}\right) \geq 0 .
\end{gathered}
$$

Then the crisp solution $X$ is obtained by $X=S^{-1} B$, where

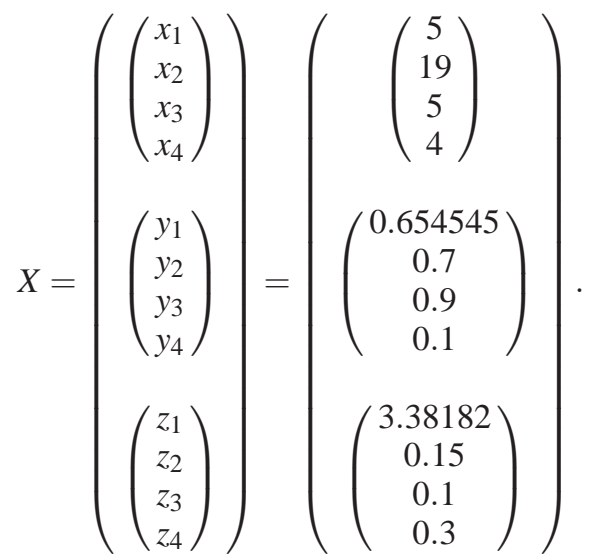

Hence the $P-X$ is

$$
\begin{gathered}
\tilde{x}_{1}=\left(x_{1}, y_{1}, z_{1}\right)=(5,0.654545,3.38182), \\
\tilde{x}_{2}=\left(x_{2}, y_{2}, z_{2}\right)=(19,0.7,0.15), \\
\tilde{x}_{3}=\left(x_{3}, y_{3}, z_{3}\right)=(5,0.9,0.1), \\
\tilde{x}_{4}=\left(x_{4}, y_{4}, z_{4}\right)=(4,0.1,0.3) .
\end{gathered}
$$

To overcome these weaknesses in Theorem 3.3., we provide the following theorem without the powerful condition $A^{-1}>0$.

Theorem 3.5. The $P-F F L S$ has $P-X$ when center matrix $A$ is invertible, that is, if

$$
\begin{aligned}
& i-\quad A^{-1}\left(I+M A^{-1}\right) b \geq A^{-1} h, \\
& i i-A^{-1} h \geq A^{-1}\left(M A^{-1}\right) b, \\
& i i i-A^{-1} g \geq A^{-1} N A^{-1} b .
\end{aligned}
$$

\section{Proof}

$y, z \geq 0$ can be easily obtained by comparing to (22), $y \geq 0$ is obtained from $A^{-1} h \geq A^{-1}\left(M A^{-1}\right) b$.

Similarly, $z \geq 0$ is obtained from $A^{-1} g \geq A^{-1} N A^{-1}$.

Now, to complete the main contribution in this theorem, we have to show $x-y \geq 0$ can be obtained without $A^{-1}>0$.

$$
\begin{gathered}
x-y=\left(A^{-1} b\right)-\left(A^{-1} h-A^{-1} M A^{-1} b\right)= \\
\left(A^{-1} b+A^{-1} M A^{-1} b\right)-\left(A^{-1} h\right),
\end{gathered}
$$

and,

$$
\begin{gathered}
\left(A^{-1} b+A^{-1} M A^{-1} b\right)=A^{-1}\left(b+M A^{-1} b\right)= \\
A^{-1}\left(I+M A^{-1}\right) b
\end{gathered}
$$

0 .

then $x-y \geq 0$ if and only if $A^{-1}\left(I+M A^{-1}\right) b-A^{-1} h \geq$

The proof is completed.

Now, we apply Theorem 3.5 for Example 3.3.

Since $A^{-1} \ngtr 0$, Theorem 3.3. fails to investigate this system:

$$
A^{-1}=\left(\begin{array}{cc}
3.1 & 2 \\
8 & 6.9
\end{array}\right)^{-1}=\left(\begin{array}{cc}
1.280 & -0.3710 \\
-1.484 & -0.3710
\end{array}\right)
$$

By applying $(i)$ in Theorem 3.5.

$$
A^{-1}\left(I+M A^{-1}\right) b=\left(\begin{array}{c}
6.64378 \\
0.152134
\end{array}\right) \text {, }
$$

and,

$$
A^{-1} h=\left(\begin{array}{c}
6.67069 \\
-2.96456
\end{array}\right)
$$

Then,

$$
A^{-1}\left(I+M A^{-1}\right) b-A^{-1} h=\left(\begin{array}{c}
-0.026901 \\
3.1167
\end{array}\right) .
$$

Hence,

$A^{-1}\left(I+M A^{-1}\right) b \ngtr A^{-1} h, \quad$ because $6.64378 \ngtr$ 6.67069 .

Finally, $P-F L L S$ does not has $P-X$.

Case 2: The conditions on $\tilde{A}$ when $\tilde{B}$ are arbitraries.

In this case the necessary and sufficient condition in positive fuzzy matrix $\tilde{A}$ to have $P-X$ when $\tilde{B}$ is an arbitrary positive fuzzy vector. (Friedman et al. [14]) investigated this case for FLS, so we will follow their technique in our case. To the best of our knowledge, this case has not been studied for FFLS.

Lemma 3.1. If $S^{-1}$ exist it must have the same structure as $S$, i.e. 


$$
S^{-1}=\left(\begin{array}{ccc}
A^{\prime} & 0 & 0 \\
M^{\prime} & A^{\prime} & 0 \\
N^{\prime} & 0 & A^{\prime}
\end{array}\right)
$$

and,

$$
\begin{gathered}
A^{\prime}=A^{-1}, \\
M^{\prime}=-A^{-1} M A^{-1} \\
N^{\prime}=-A^{-1} N A^{-1} .
\end{gathered}
$$

\section{Proof}

Let $(S: I)$ be a rectangle block matrix,

$$
(S: I)=\left(\begin{array}{ccccccc}
A & 0 & 0 & \vdots & I & 0 & 0 \\
M & A & 0 & \vdots & 0 & I & 0 \\
N & 0 & A & & 0 & 0 & I \\
& & \vdots & &
\end{array}\right),
$$

using Theorem 3.1., $S^{-1}$ is exist then $A^{-1}$ is exist. By multiplying each rows by $A^{-1}$,

$$
\left(\begin{array}{cccccccc}
I & 0 & 0 & \vdots & A^{-1} & 0 & 0 \\
A^{-1} M & I & 0 & \vdots & 0 & A^{-1} & 0 \\
A^{-1} N & 0 & I & & 0 & 0 & A^{-1}
\end{array}\right),
$$

0.

Lemma 3.2. The matrix $S$ is an inverse-nonnegative, if and only if the centric matrix $A$ be an inverse-nonnegative and the spreads matrices $M$ and $N$ are zero matrices, i.e. $S^{-1}>0$, if and only if $A^{-1}>0$, and $\tilde{A}=(A, 0,0)$.

\section{Proof}

By Lemma 3.1.

$$
S^{-1}=\left(\begin{array}{ccc}
A^{\prime} & 0 & 0 \\
M^{\prime} & A^{\prime} & 0 \\
N^{\prime} & 0 & A^{\prime}
\end{array}\right)
$$

$$
=\left(\begin{array}{ccc}
A^{-1} & 0 & 0 \\
-A^{-1} M A^{-1} & A^{-1} & 0 \\
-A^{-1} N A^{-1} & 0 & A^{-1}
\end{array}\right),
$$

$A^{\prime}>0$ if and only if $A^{-1}>0$.

$M^{\prime} \geq 0$ if and only if $-A^{-1} M A^{-1} \geq 0$ implies that $M=$ $N^{\prime} \geq 0$ if and only if $-A^{-1} N A^{-1} \geq 0$ implies that $N=$ 0 .

The proof is completed.

The following result answers case 2 .

Theorem 3.6. If $A^{-1}>0$ and the spreads matrices $M, N$ are zeros, then the unique solution $X$ of $S X=B$ represents a positive $L R$ fuzzy number vector $\tilde{X}$ for an arbitrary positive $L R$ fuzzy number vector $\tilde{B}$ in $\tilde{A} \otimes X=\tilde{B}$.

\section{Proof}

$\tilde{B} \geq 0$ by hypothesis implies $B \geq 0$.

By Lemma 3.1.,

$$
A^{-1}>0 \text { and } M=N=0 \text { then } S^{-1}>0 .
$$

Hence,

$$
S^{-1} B \geq 0 \Longrightarrow X \geq 0 \Longrightarrow y, z \geq 0 .
$$

Then $\tilde{X}$ is LR fuzzy vector.

Now the nonnegative of $\tilde{X}$ is obtained as follows:

$$
\begin{gathered}
x-y=A^{-1} b-A^{-1} h+A^{-1} M A^{-1} b, \\
=A^{-1}(b-h), \text { since } b \geq h, \\
\text { then } x-y \geq 0 .
\end{gathered}
$$

$$
\begin{aligned}
& M^{\prime}=-A^{-1} M A^{-1}, \\
& N^{\prime}=-A^{-1} N A^{-1} .
\end{aligned}
$$


This theorem shows that the conditions in $\tilde{A}=(A, M, N)$ to let $\tilde{B}$ an arbitrary positive vector are $A^{-1}>0$ and $M=N=0$ in $\tilde{A}=(A, M, N)$.

Corollary 3.3. The unique solution $X$ of $S X=B$ represents a positive $L R$ fuzzy number vector for an arbitrary a positive LR fuzzy number vector $\tilde{B}$ if

$$
\begin{aligned}
& i-A^{-1}>0, \\
& i i-\text { The FFLS is a LR-FLS. }
\end{aligned}
$$

\section{Proof}

According to previous Theorem 3.6.,

$$
M=N=0 \text { then } \tilde{A}=(A, 0,0) .
$$

Hence the fuzzy matrix $\tilde{A}$ represents by only crisp matrix $A$.

Unfortunately, the guarantee of $A^{-1}$ to be nonnegative is very small as is shown in Theorem 3.4.

Now we illustrate an example for this case under the previous conditions.

Example 3.5. Consider the following FFLS. Find the positive solution, when $\tilde{B}$ is an arbitrary positive LR fuzzy vector.

$$
\left\{\begin{array}{l}
(3,0,0) \otimes\left(x_{1}, y_{1}, z_{1}\right)=\left(b_{1}, h_{1}, g_{1}\right), \\
(4,0,0) \otimes\left(x_{2}, y_{2}, z_{2}\right)=\left(b_{2}, h_{2}, g_{2}\right), \\
(7,0,0) \otimes\left(x_{3}, y_{3}, z_{3}\right)=\left(b_{3}, h_{3}, g_{3}\right) .
\end{array}\right.
$$

Where $\tilde{x}_{i}=\left(x_{i}, y_{i}, z_{i}\right) \geq 0, i=1,2,3$.

\section{Solution}

The system may be written in matrix form,

$$
\begin{gathered}
\left(\begin{array}{c}
(0,0,0)(0,0,0)(3,0,0) \\
(4,0,0)(0,0,0)(0,0,0) \\
(0,0,0)(7,0,0)(0,0,0)
\end{array}\right) \\
\left(\begin{array}{l}
\left(x_{1}, y_{1}, z_{1}\right) \\
\left(x_{2}, y_{2}, z_{2}\right) \\
\left(x_{3}, y_{3}, z_{3}\right)
\end{array}\right)=\left(\begin{array}{l}
\left(b_{1}, h_{1}, g_{1}\right) \\
\left(b_{2}, h_{2}, g_{2}\right) \\
\left(b_{3}, h_{3}, g_{3}\right)
\end{array}\right) .
\end{gathered}
$$

The associated linear system $S X=B$ can be obtained as follows:

$$
\left(\begin{array}{lllllllll}
0 & 0 & 3 & 0 & 0 & 0 & 0 & 0 & 0 \\
4 & 0 & 0 & 0 & 0 & 0 & 0 & 0 & 0 \\
0 & 7 & 0 & 0 & 0 & 0 & 0 & 0 & 0 \\
0 & 0 & 0 & 0 & 0 & 3 & 0 & 0 & 0 \\
0 & 0 & 0 & 4 & 0 & 0 & 0 & 0 & 0 \\
0 & 0 & 0 & 0 & 7 & 0 & 0 & 0 & 0 \\
0 & 0 & 0 & 0 & 0 & 0 & 0 & 0 & 3 \\
0 & 0 & 0 & 0 & 0 & 0 & 4 & 0 & 0 \\
0 & 0 & 0 & 0 & 0 & 0 & 0 & 7 & 0
\end{array}\right)
$$

$$
\left(\begin{array}{l}
x_{1} \\
x_{2} \\
x_{3} \\
y_{1} \\
y_{2} \\
y_{3} \\
z_{1} \\
z_{2} \\
z_{3}
\end{array}\right)=\left(\begin{array}{l}
b_{1} \\
b_{2} \\
b_{3} \\
h_{1} \\
h_{2} \\
h_{3} \\
g_{1} \\
g_{2} \\
g_{3}
\end{array}\right) .
$$

By computing $S^{-1} B$, then

$$
X=\left(\begin{array}{c}
x_{1} \\
x_{2} \\
x_{3} \\
y_{1} \\
y_{2} \\
y_{3} \\
z_{1} \\
z_{2} \\
z_{3}
\end{array}\right)=\left(\begin{array}{c}
\frac{b_{2}}{4} \\
\frac{b_{3}}{7} \\
\frac{b_{1}}{3} \\
\frac{h_{2}}{4} \\
\frac{h_{3}}{7} \\
\frac{h_{1}}{3} \\
\frac{g_{2}}{4} \\
\frac{g_{3}}{7} \\
\frac{g_{1}}{3}
\end{array}\right) .
$$

The general solution for an arbitrary fuzzy vector $\tilde{B}$ is

$$
\begin{aligned}
& \tilde{x}_{1}=\left(x_{1}, y_{1}, z_{1}\right)=\left(\frac{b_{2}}{4}, \frac{h_{2}}{4}, \frac{g_{2}}{4}\right), \\
& \tilde{x}_{2}=\left(x_{2}, y_{2}, z_{2}\right)=\left(\frac{b_{3}}{7}, \frac{h_{3}}{7}, \frac{g_{3}}{7}\right), \\
& \tilde{x}_{3}=\left(x_{3}, y_{3}, z_{3}\right)=\left(\frac{b_{1}}{3}, \frac{h_{1}}{3}, \frac{g_{1}}{3}\right) .
\end{aligned}
$$

Some particular solutions of this FFLS are:

-When

$$
B=\left(\begin{array}{c}
\left(b_{1}, h_{1}, g_{1}\right) \\
\left(b_{2}, h_{2}, g_{2}\right) \\
\left(b_{3}, h_{3}, g_{3}\right)
\end{array}\right)=\left(\begin{array}{c}
(5,3,8) \\
(3,2,1) \\
(4,3,1)
\end{array}\right)
$$

then

$$
\tilde{X}=\left(\begin{array}{l}
\left(x_{1}, y_{1}, z_{1}\right) \\
\left(x_{2}, y_{2}, z_{2}\right) \\
\left(x_{3}, y_{3}, z_{3}\right)
\end{array}\right)=\left(\begin{array}{c}
\left(\frac{3}{4}, \frac{1}{2}, \frac{1}{4}\right) \\
\left(\frac{4}{7}, \frac{3}{7}, \frac{1}{7}\right) \\
\left(\frac{5}{3}, 1, \frac{8}{3}\right)
\end{array}\right) .
$$


-When

$$
B=\left(\begin{array}{l}
\left(b_{1}, h_{1}, g_{1}\right) \\
\left(b_{2}, h_{2}, g_{2}\right) \\
\left(b_{3}, h_{3}, g_{3}\right)
\end{array}\right)=\left(\begin{array}{l}
(7,2,1) \\
(6,0,1) \\
(3,1,9)
\end{array}\right)
$$

then

$$
\tilde{X}=\left(\begin{array}{l}
\left(x_{1}, y_{1}, z_{1}\right) \\
\left(x_{2}, y_{2}, z_{2}\right) \\
\left(x_{3}, y_{3}, z_{3}\right)
\end{array}\right)=\left(\begin{array}{c}
\left(\frac{3}{2}, 0, \frac{1}{4}\right) \\
\left(\frac{3}{7}, \frac{1}{7}, \frac{9}{7}\right) \\
\left(\frac{7}{3}, \frac{2}{3}, \frac{1}{3}\right)
\end{array}\right) .
$$

-When

$$
B=\left(\begin{array}{l}
\left(b_{1}, h_{1}, g_{1}\right) \\
\left(b_{2}, h_{2}, g_{2}\right) \\
\left(b_{3}, h_{3}, g_{3}\right)
\end{array}\right)=\left(\begin{array}{l}
(1,1,1) \\
(3,3,3) \\
(6,6,6)
\end{array}\right)
$$

then

$$
\tilde{X}=\left(\begin{array}{l}
\left(x_{1}, y_{1}, z_{1}\right) \\
\left(x_{2}, y_{2}, z_{2}\right) \\
\left(x_{3}, y_{3}, z_{3}\right)
\end{array}\right)=\left(\begin{array}{c}
\left(\frac{3}{4}, \frac{3}{4}, \frac{3}{4}\right) \\
\left(\frac{6}{7}, \frac{6}{7}, \frac{6}{7}\right) \\
\left(\frac{1}{3}, \frac{1}{3}, \frac{1}{3}\right)
\end{array}\right) .
$$

We note that the solution must be positive LR fuzzy vector for any arbitrary right hand side positive fuzzy vector $\tilde{B}$.

\subsection{Solution of LR-FLS}

In this section we will show the $P-X$ for positive LR FLS $(P-L R-F L S)$ can be obtained by supposing the spreads matrices are zero, i.e. $M=N=0$ in

$$
\left(\begin{array}{lll}
A & 0 & 0 \\
0 & A & 0 \\
0 & 0 & A
\end{array}\right)\left(\begin{array}{l}
x \\
y \\
z
\end{array}\right)=\left(\begin{array}{l}
b \\
h \\
g
\end{array}\right) .
$$

The solution of $P-L R-F L S$ can computed easily or directly by

$$
\left\{\begin{array}{l}
x=A^{-1} b \\
y=A^{-1} h \\
z=A^{-1} g .
\end{array}\right.
$$

A necessary and sufficient condition For $P-L R-F L S$ is needed, and according to Corollary 3.3. this method can obtain the solution for an arbitrary vector positive $\tilde{B}$ as is shown in Example 3.5. Other examples for $P-L R-F L S$ are illustrated in Example 3.6. and for $10 \times 10$ FFLS in Example 3.7.

Example 3.6. Find $P-X$ for the following $L R-F L S$.

$$
\left\{\begin{aligned}
10 x_{1}+9 x_{2} & =(120,4,9), \\
x_{1}+8 x_{2} & =(80,1,5) .
\end{aligned}\right.
$$

Where $\tilde{x}_{i}=\left(x_{i}, y_{i}, z_{i}\right) \geq 0, i=1,2$.

\section{Solution}

The system may be written in FFLS form,

$(10,0,0) \otimes\left(x_{1}, y_{1}, z_{1}\right) \oplus(9,0,0) \otimes\left(x_{2}, y_{2}, z_{2}\right)=$ $(120,4,9)$,

$(1,0,0) \otimes\left(x_{1}, y_{1}, z_{1}\right) \oplus(8,0,0) \otimes\left(x_{2}, y_{2}, z_{2}\right)=$ $(80,1,5)$.

Then $S X=B$ is

$$
\left(\begin{array}{cccccc}
10 & 9 & 0 & 0 & 0 & 0 \\
1 & 8 & 0 & 0 & 0 & 0 \\
0 & 0 & 10 & 9 & 0 & 0 \\
0 & 0 & 1 & 8 & 0 & 0 \\
0 & 0 & 0 & 0 & 10 & 9 \\
0 & 0 & 0 & 0 & 1 & 8
\end{array}\right)\left(\begin{array}{l}
x_{1} \\
x_{2} \\
y_{1} \\
y_{2} \\
z_{1} \\
z_{2}
\end{array}\right)=\left(\begin{array}{c}
120 \\
80 \\
4 \\
1 \\
9 \\
5
\end{array}\right) .
$$

The crisp solution $X$ is obtained by $X=S^{-1} B$,

$$
X=\left(\begin{array}{c}
x_{1} \\
x_{2} \\
y_{1} \\
y_{2} \\
z_{1} \\
z_{2}
\end{array}\right)=\left(\begin{array}{c}
\frac{240}{71} \\
\frac{680}{71} \\
\frac{23}{71} \\
\frac{6}{71} \\
\frac{27}{71} \\
\frac{41}{71}
\end{array}\right) .
$$

Hence the positive solution is

$$
\begin{gathered}
\tilde{x}_{1}=\left(x_{1}, y_{1}, z_{1}\right)=\left(\frac{240}{71}, \frac{23}{71}, \frac{27}{71}\right), \\
\tilde{x}_{2}=\left(x_{2}, y_{2}, z_{2}\right)=\left(\frac{680}{71}, \frac{6}{71}, \frac{41}{71}\right) .
\end{gathered}
$$


Example 3.7. Find $P-X$ for the following $10 \times 10$ FFLS.

$(5,0,1) \otimes\left(x_{1}, y_{1}, z_{1}\right) \oplus(6,3,0) \otimes\left(x_{2}, y_{2}, z_{2}\right) \oplus$ $(2,2,1) \otimes\left(x_{3}, y_{3}, z_{3}\right) \oplus(3,2,2) \otimes\left(x_{4}, y_{4}, z_{4}\right) \oplus(4,1,3) \otimes$ $\left(x_{5}, y_{5}, z_{5}\right) \oplus(8,3,4) \otimes\left(x_{6}, y_{6}, z_{6}\right) \oplus(7,0,5) \otimes$ $\left(x_{7}, y_{7}, z_{7}\right) \oplus(9,2,6) \otimes\left(x_{8}, y_{8}, z_{8}\right) \oplus(8,3,4) \otimes$ $\left(x_{9}, y_{9}, z_{9}\right) \oplus(9,1,5) \otimes\left(x_{10}, y_{10}, z_{10}\right)=(450,297,473)$,

$(4,3,0) \otimes\left(x_{1}, y_{1}, z_{1}\right) \oplus(4,1,8) \otimes\left(x_{2}, y_{2}, z_{2}\right) \oplus$ $(6,2,8) \otimes\left(x_{3}, y_{3}, z_{3}\right) \oplus(7,1,3) \otimes\left(x_{4}, y_{4}, z_{4}\right) \oplus(8,0,6) \otimes$ $\left(x_{5}, y_{5}, z_{5}\right) \oplus(4,1,7) \otimes\left(x_{6}, y_{6}, z_{6}\right) \oplus(5,2,6) \otimes$ $\left(x_{7}, y_{7}, z_{7}\right) \oplus(9,1,3) \otimes\left(x_{8}, y_{8}, z_{8}\right) \oplus(7,3,5) \otimes$ $\left(x_{9}, y_{9}, z_{9}\right) \oplus(8,3,1) \otimes\left(x_{10}, y_{10}, z_{10}\right)=(476,303,548)$,

$(2,2,3) \otimes\left(x_{1}, y_{1}, z_{1}\right) \oplus(7,3,5) \otimes\left(x_{2}, y_{2}, z_{2}\right) \oplus$ $(6,4,1) \otimes\left(x_{3}, y_{3}, z_{3}\right) \oplus(4,2,2) \otimes\left(x_{4}, y_{4}, z_{4}\right) \oplus(4,0,2) \otimes$ $\left(x_{5}, y_{5}, z_{5}\right) \oplus(3,1,5) \otimes\left(x_{6}, y_{6}, z_{6}\right) \oplus(4,4,8) \otimes$ $\left(x_{7}, y_{7}, z_{7}\right) \oplus(5,2,8) \otimes\left(x_{8}, y_{8}, z_{8}\right) \oplus(6,2,3) \otimes$ $\left(x_{9}, y_{9}, z_{9}\right) \oplus(7,2,2) \otimes\left(x_{10}, y_{10}, z_{10}\right)=(359,293,452)$,

$(3,2,6) \otimes\left(x_{1}, y_{1}, z_{1}\right) \oplus(6,1,3) \otimes\left(x_{2}, y_{2}, z_{2}\right) \oplus$ $(8,0,0) \otimes\left(x_{3}, y_{3}, z_{3}\right) \oplus(5,2,1) \otimes\left(x_{4}, y_{4}, z_{4}\right) \oplus(8,4,1) \otimes$ $\left(x_{5}, y_{5}, z_{5}\right) \oplus(8,1,5) \otimes\left(x_{6}, y_{6}, z_{6}\right) \oplus(4,4,8) \otimes$ $\left(x_{7}, y_{7}, z_{7}\right) \oplus(5,2,8) \otimes\left(x_{8}, y_{8}, z_{8}\right) \oplus(6,2,3) \otimes$ $\left(x_{9}, y_{9}, z_{9}\right) \oplus(7,2,2) \otimes\left(x_{10}, y_{10}, z_{10}\right)=(359,293,452)$,

$(4,3,8) \otimes\left(x_{1}, y_{1}, z_{1}\right) \oplus(3,2,9) \otimes\left(x_{2}, y_{2}, z_{2}\right) \oplus$ $(2,1,3) \otimes\left(x_{3}, y_{3}, z_{3}\right) \oplus(1,0,0) \otimes\left(x_{4}, y_{4}, z_{4}\right) \oplus(3,1,5) \otimes$ $\left(x_{5}, y_{5}, z_{5}\right) \oplus(7,6,2) \otimes\left(x_{6}, y_{6}, z_{6}\right) \oplus(6,0,2) \otimes$ $\left(x_{7}, y_{7}, z_{7}\right) \oplus(3,1,8) \otimes\left(x_{8}, y_{8}, z_{8}\right) \oplus(4,2,6) \otimes$ $\left(x_{9}, y_{9}, z_{9}\right) \oplus(5,2,9) \otimes\left(x_{10}, y_{10}, z_{10}\right)=(279,236,562)$,

$(5,1,4) \otimes\left(x_{1}, y_{1}, z_{1}\right) \oplus(4,4,8) \otimes\left(x_{2}, y_{2}, z_{2}\right) \oplus$ $(3,2,8) \otimes\left(x_{3}, y_{3}, z_{3}\right) \oplus(2,1,3) \otimes\left(x_{4}, y_{4}, z_{4}\right) \oplus(8,5,4) \otimes$ $\left(x_{5}, y_{5}, z_{5}\right) \oplus(6,2,8) \otimes\left(x_{6}, y_{6}, z_{6}\right) \oplus(3,1,5) \otimes$ $\left(x_{7}, y_{7}, z_{7}\right) \oplus(2,2,3) \otimes\left(x_{8}, y_{8}, z_{8}\right) \oplus(3,2,2) \otimes$ $\left(x_{9}, y_{9}, z_{9}\right) \oplus(4,3,5) \otimes\left(x_{10}, y_{10}, z_{10}\right)=(300,280,519)$,

$(6,0,3) \otimes\left(x_{1}, y_{1}, z_{1}\right) \oplus(5,2,1) \otimes\left(x_{2}, y_{2}, z_{2}\right) \oplus$ $(7,3,4) \otimes\left(x_{3}, y_{3}, z_{3}\right) \oplus(3,2,4) \otimes\left(x_{4}, y_{4}, z_{4}\right) \oplus(8,1,3) \otimes$ $\left(x_{5}, y_{5}, z_{5}\right) \oplus(5,3,1) \otimes\left(x_{6}, y_{6}, z_{6}\right) \oplus(4,3,8) \otimes$ $\left(x_{7}, y_{7}, z_{7}\right) \oplus(7,5,0) \otimes\left(x_{8}, y_{8}, z_{8}\right) \oplus(2,1,3) \otimes$ $\left(x_{9}, y_{9}, z_{9}\right) \oplus(3,1,3) \otimes\left(x_{10}, y_{10}, z_{10}\right)=(384,294,423)$,

$(7,5,1) \otimes\left(x_{1}, y_{1}, z_{1}\right) \oplus(6,5,4) \otimes\left(x_{2}, y_{2}, z_{2}\right) \oplus$ $(5,4,5) \otimes\left(x_{3}, y_{3}, z_{3}\right) \oplus(4,1,8) \otimes\left(x_{4}, y_{4}, z_{4}\right) \oplus(3,2,8) \otimes$ $\left(x_{5}, y_{5}, z_{5}\right) \oplus(7,1,3) \otimes\left(x_{6}, y_{6}, z_{6}\right) \oplus(6,2,8) \otimes$ $\left(x_{7}, y_{7}, z_{7}\right) \oplus(9,3,1) \otimes\left(x_{8}, y_{8}, z_{8}\right) \oplus(7,5,4) \otimes$ $\left(x_{9}, y_{9}, z_{9}\right) \oplus(9,2,7) \otimes\left(x_{10}, y_{10}, z_{10}\right)=(471,406,628)$,
$(8,2,7) \otimes\left(x_{1}, y_{1}, z_{1}\right) \oplus(7,2,5) \otimes\left(x_{2}, y_{2}, z_{2}\right) \oplus$ $(6,4,1) \otimes\left(x_{3}, y_{3}, z_{3}\right) \oplus(5,2,5) \otimes\left(x_{4}, y_{4}, z_{4}\right) \oplus(4,3,7) \otimes$ $\left(x_{5}, y_{5}, z_{5}\right) \oplus(9,2,8) \otimes\left(x_{6}, y_{6}, z_{6}\right) \oplus(8,3,3) \otimes$ $\left(x_{7}, y_{7}, z_{7}\right) \oplus(7,6,0) \otimes\left(x_{8}, y_{8}, z_{8}\right) \oplus(8,4,2) \otimes$ $\left(x_{9}, y_{9}, z_{9}\right) \oplus(7,3,1) \otimes\left(x_{10}, y_{10}, z_{10}\right)=(508,432,565)$,

$(9,3,8) \otimes\left(x_{1}, y_{1}, z_{1}\right) \oplus(8,2,4) \otimes\left(x_{2}, y_{2}, z_{2}\right) \oplus$ $(7,4,9) \otimes\left(x_{3}, y_{3}, z_{3}\right) \oplus(6,1,8) \otimes\left(x_{4}, y_{4}, z_{4}\right) \oplus(5,2,8) \otimes$ $\left(x_{5}, y_{5}, z_{5}\right) \oplus(4,3,8) \otimes\left(x_{6}, y_{6}, z_{6}\right) \oplus(3,2,8) \otimes$ $\left(x_{7}, y_{7}, z_{7}\right) \oplus(9,1,3) \otimes\left(x_{8}, y_{8}, z_{8}\right) \oplus(6,4,1) \otimes$ $\left(x_{9}, y_{9}, z_{9}\right) \oplus(6,1,3) \otimes\left(x_{10}, y_{10}, z_{10}\right)=(477,327,700)$.

Where $\tilde{x}_{i}=\left(x_{i}, y_{i}, z_{i}\right) \geq 0, i=1, \ldots, 10$.

\section{Solution}

The system may be written in matrix form,

$$
\left(\begin{array}{lll}
A & 0 & 0 \\
M & A & 0 \\
N & 0 & A
\end{array}\right)\left(\begin{array}{l}
x \\
y \\
z
\end{array}\right)=\left(\begin{array}{l}
b \\
h \\
g
\end{array}\right) .
$$

where,

$$
A=\left(\begin{array}{llllllllll}
5 & 6 & 2 & 3 & 4 & 8 & 7 & 9 & 8 & 9 \\
4 & 4 & 6 & 7 & 8 & 4 & 5 & 9 & 7 & 8 \\
2 & 7 & 6 & 4 & 4 & 3 & 4 & 5 & 6 & 7 \\
3 & 6 & 8 & 5 & 8 & 8 & 5 & 4 & 5 & 6 \\
4 & 3 & 2 & 1 & 3 & 7 & 6 & 3 & 4 & 5 \\
5 & 4 & 3 & 2 & 8 & 6 & 3 & 2 & 3 & 4 \\
6 & 5 & 7 & 3 & 8 & 5 & 4 & 7 & 2 & 3 \\
7 & 6 & 5 & 4 & 3 & 7 & 6 & 9 & 7 & 9 \\
8 & 7 & 6 & 5 & 4 & 9 & 8 & 7 & 8 & 7 \\
9 & 8 & 7 & 6 & 5 & 4 & 3 & 9 & 6 & 6
\end{array}\right),
$$

$$
M=\left(\begin{array}{llllllllll}
0 & 3 & 2 & 2 & 1 & 3 & 0 & 2 & 3 & 1 \\
3 & 1 & 2 & 1 & 0 & 1 & 2 & 1 & 3 & 3 \\
2 & 3 & 4 & 2 & 0 & 1 & 4 & 2 & 2 & 2 \\
2 & 1 & 0 & 2 & 4 & 1 & 1 & 1 & 3 & 3 \\
3 & 2 & 1 & 0 & 1 & 6 & 0 & 1 & 2 & 2 \\
1 & 4 & 2 & 1 & 5 & 2 & 1 & 2 & 2 & 3 \\
0 & 2 & 3 & 2 & 1 & 3 & 3 & 5 & 1 & 1 \\
5 & 5 & 4 & 1 & 2 & 1 & 2 & 3 & 5 & 2 \\
2 & 2 & 4 & 2 & 3 & 2 & 3 & 6 & 4 & 3 \\
3 & 2 & 4 & 1 & 2 & 3 & 2 & 1 & 4 & 1
\end{array}\right),
$$

$$
N=\left(\begin{array}{llllllllll}
1 & 0 & 1 & 2 & 3 & 4 & 5 & 6 & 4 & 5 \\
0 & 8 & 8 & 3 & 6 & 7 & 6 & 3 & 5 & 1 \\
3 & 5 & 1 & 2 & 2 & 5 & 8 & 8 & 3 & 2 \\
6 & 3 & 0 & 1 & 1 & 5 & 4 & 7 & 2 & 7 \\
8 & 9 & 3 & 0 & 5 & 2 & 2 & 8 & 6 & 9 \\
4 & 8 & 8 & 3 & 4 & 8 & 5 & 3 & 2 & 5 \\
3 & 1 & 4 & 4 & 3 & 1 & 8 & 0 & 3 & 3 \\
1 & 4 & 5 & 8 & 8 & 3 & 8 & 1 & 4 & 7 \\
7 & 5 & 1 & 5 & 7 & 8 & 3 & 0 & 2 & 1 \\
8 & 4 & 9 & 8 & 8 & 8 & 8 & 3 & 1 & 3
\end{array}\right)
$$




$$
b=\left(\begin{array}{l}
450 \\
476 \\
359 \\
427 \\
279 \\
300 \\
384 \\
471 \\
508 \\
477
\end{array}\right), h=\left(\begin{array}{l}
297 \\
303 \\
293 \\
293 \\
236 \\
280 \\
294 \\
406 \\
432 \\
327
\end{array}\right), g=\left(\begin{array}{l}
473 \\
548 \\
452 \\
480 \\
562 \\
519 \\
423 \\
628 \\
565 \\
700
\end{array}\right) .
$$$$
x=\left(\begin{array}{l}
x_{1} \\
x_{2} \\
x_{3} \\
x_{4} \\
x_{5} \\
x_{6} \\
x_{7} \\
x_{8} \\
x_{9} \\
x_{10}
\end{array}\right), y=\left(\begin{array}{l}
y_{1} \\
y_{2} \\
y_{3} \\
y_{4} \\
y_{5} \\
y_{6} \\
y_{7} \\
y_{8} \\
y_{9} \\
y_{10}
\end{array}\right), z=\left(\begin{array}{l}
z_{1} \\
z_{2} \\
z_{3} \\
z_{4} \\
z_{5} \\
z_{6} \\
z_{7} \\
z_{8} \\
z_{9} \\
z_{10}
\end{array}\right) .
$$

Then the crisp solution $X$ is obtained by $X=S^{-1} B$,

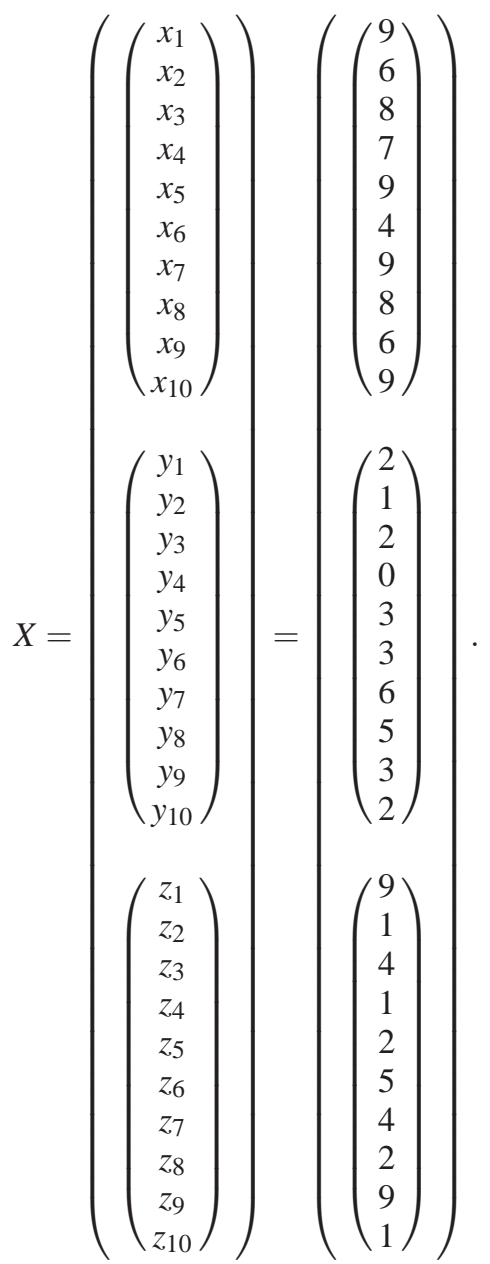

Then the fuzzy solution is

$$
\tilde{X}=\left(\begin{array}{l}
\left(x_{1}, y_{1}, z_{1}\right) \\
\left(x_{2}, y_{2}, z_{2}\right) \\
\left(x_{3}, y_{3}, z_{3}\right) \\
\left(x_{4}, y_{4}, z_{4}\right) \\
\left(x_{5}, y_{5}, z_{5}\right) \\
\left(x_{6}, y_{6}, z_{6}\right) \\
\left(x_{7}, y_{7}, z_{7}\right) \\
\left(x_{8}, y_{8}, z_{8}\right) \\
\left(x_{9}, y_{9}, z_{9}\right) \\
\left(x_{10}, y_{10}, z_{10}\right)
\end{array}\right)=\left(\begin{array}{l}
(9,2,9) \\
(6,1,1) \\
(8,2,4) \\
(7,0,1) \\
(9,3,2) \\
(4,3,5) \\
(9,6,4) \\
(8,5,2) \\
(6,3,9) \\
(9,2,1)
\end{array}\right) .
$$

\section{Conclusion}

Positive solution of fully fuzzy linear systems, where the coefficients are postive, can be solved by classical methods of linear algebra through Gauss elimination method, Cramer's rule, Cholesky method, decomposition method, and some other iterative methods. These computational methods have many disadvantages such as the large number of iterations, finding more numbers of determinants or inverse of many matrices, and large number of computational steps. In order to solve the fully fuzzy linear system, we proposed a model based on the representation of all matrices in one block matrix then making two computational steps by finding the inverse of the block matrix. After that, the method was used to solve fully fuzzy linear systems and the consistency of fuzzy solution can be checked by an associated linear system. The method was employed to solve LR-FLS.

\section{References}

[1] A. Kaufmann and M. M. Gupta, Introduction to Fuzzy, New York, NY, USA: Van Nostrand Reinhold, (1991).

[2] A. Kumar, A. Bansal and Neetu, Solution of fully fuzzy linear system with arbitrary coefficients, International Journal of Alied Mathematics and Computation, 3, 232-237 (2011).

[3] A. Kumar, Neetu and A. Bansal, A New Aroach for Solving Fully Fuzzy Linear Systems, Hindawi Publishing Corporation, 1-8 (2011).

[4] A. Kumar, Neetu and A. Bansal, A New Computational Method for Solving Fully Fuzzy Linear Systems of Triangular Fuzzy Numbers, Fuzzy Inf. Eng., 63-73 (2012). 
[5] D. Dubois and H. Prade, Operations on fuzzy numbers, International Journal of Systems Science, 9, 613-626 (1978).

[6] F. Zhang, Matrix Theory:Basic Results and Techniques, Linear Park, Davie, Florida, USA, (2010).

[7] H.-K. Liu, On the solution of fully fuzzy linear systems, International Journal of Computational and Mathematical Sciences, 29-33 (2010).

[8] J. Buckley and Y. Qu, Solving systems of linear fuzzy equations, Fuzzy Sets and Systems, 43, 33-43 (1991).

[9] L. A. Zadeh, Fuzzy sets., Information and Control, 8, 338353 (1965).

[10] L. A. Zadeh, The Concept of a Linguistic Variable and its Alication to Aroximate Reasoning-II, INFORMATION SCIENCES, 8, 301-357 (1975).

[11] M. Dehghan and B. Hashemi, Solution of the fully fuzzy linear systems using the decomposition procedure, Alied Mathematics and Computation, 182, 1568-1580 (2006).

[12] M. Dehghan, B. Hashemi and M. Ghatee, Computational methods for solving fully fuzzy linear systems, Alied Mathematics and Computation, 179, 328-343 (2006).

[13] M. Dehghan, B. Hashemi and M. Ghatee, Solution of the fully fuzzy linear systems using iterative techniques, Chaos, Solitons and Fractals, 34, 316-336 (2007).

[14] M. Friedman, M. Ming and A. Kandel, Fuzzy linear systems, Fuzzy Sets and Systems, 96, 201-209 (1998).

[15] M. Mosleh, M. Otadi and A. Khanmirzaie, Decomposition Method for Solving Fully Fuzzy Linear Systems, Iranian Journal of Optimization, 188-198 (2009).

[16] M. Otadi and M. Mosleh, Solving fully fuzzy matrix equations, Alied Mathematical Modelling, 36, 6114-6121 (2012).

[17] M. Otadi, M. Mosleh and S. Abbasbandy, Numerical solution of fully fuzzy linear systems by fuzzy neural network, Soft Comput, 1513-1522 (2011).

[18] Ming, M.; Friedman, M.; Kandel, A., General fuzzy least squares, Fuzzy Sets and Systems, 88, 107-118 (1997).

[19] N. Babbar, A. Kumar and A. Bansal, Solving fully fuzzy linear system with arbitrary triangular fuzzy numbers $(m, \alpha, \beta)$, Soft Comput, 1-12 (2012).

[20] R. Ezzati, S. Khezerloo and A. Yousefzadeh, Solving Fully Fuzzy Linear System of Equations in General Form, Journal of Fuzzy Set Valued Analysis, 1-11 (2012).

[21] S. Abbasbandy and M. S. Hashemi, Solving Fully Fuzzy Linear Systems by Using Implicit Gauss-Cholesky Algorithm, Computational Mathematics and Modeling, 1, 535-541 (2012).

[22] S. H. Nasseri and F. Zahmatkesh, Huang method for solving fully fuzzy linear system of equations, Journal of Mathematics and Computer Science, 1, 1-5 (2010).

[23] S. H. Nasseri, M. Sohrabi and E. Ardil, Solving Fully Fuzzy Linear Systems by use of a Certain Decomposition of the Coefficient Matrix, International Journal of Computational and Mathematical Sciences, 3, 140-142 (2008).

[24] S. Nasseri, M. Matinfar and Z. Kheiri, Greville's method for the fully fuzzy linear system of equations, Adv Fuzzy Sets Syst, 4, 301-3011 (2009).

[25] S. Nasseri and M. Sohrabi, Cholesky Decomposition For Solving The Fully Fuzzy Linear System Of Equations, International Journal of Alied Mathematics, 22, 689-696 (2009).
[26] S. Muzziolia and H. Reynaertsb, Fuzzy linear systems of the form $A_{1} x+b_{1}=A_{2} x+b_{2}$, Fuzzy Sets and Systems, 157, 939-951 (2006).

[27] T. Allahviranloo, M. Ghanbari and A. Hosseinzadeh, A note on "Fuzzy linear systems", Fuzzy Sets and Systems, 177, 87-92 (2011).

[28] T. Allahviranloo, N. Mikaeilvand, F. H. Lotfi and M. F. Jelodar, Fully Fuzzy Linear Systems, International Journal of Alied Operational Research, 1, 35-48 (2011).

[29] T. Allahviranloo, N. Mikaeilvand, N. A. Kiani and R. M. Shabestari, Signed Decomposition of Fully Fuzzy Linear Systems, Al. Al. Math., 3, 77-88 (2008).

[30] T. Allahviranloo, R. Nuraei, M. Ghanbari, E. Haghi and A. A. Hosseinzadeh, A new metric for L-R fuzzy numbers and its alication in fuzzy linear systems, Soft Comput, 17431754 (2012).

[31] T. Allahviranlooa, S. Salahshourb and M. Khezerlooa, Maximal- and minimal symmetric solutions of fully fuzzy linear systems, Journal of Computational and Alied Mathematics, 235, 4652-4662 (2011).

[32] H. Minc Nonnegative Matrices, John Wiley and Sons, New York, (1988).

[33] G. Malkawi, N. Ahmad and H. Ibrahim, A note on "Solving fully fuzzy linear systems by using implicit gausscholesky algorithm”, Comput. Math. Model, (2013) (accepted).

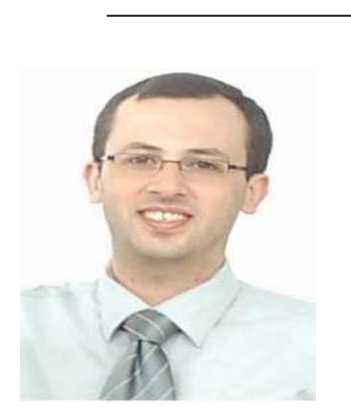
Ghassan Malkawi
received his MS degree
in applied mathematics
from University of Jordan
in 2004. Currently, he
is a PhD researcher under
the supervising of Dr.
Nazihah Ahmad and Haslinda
Ibrahim in University Utara
- Malaysia. Mr. Malkawi's research interests are focusing on Fuzzy Mathematics.

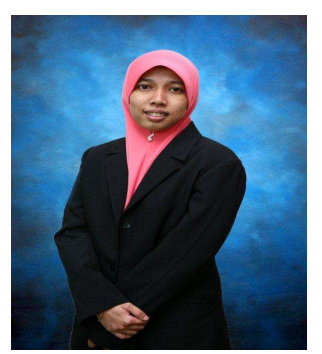

\section{Nazihah}

Ahmad is a senior lecturer at School of Quantitative Sciences, Universiti Utara Malaysia. She obtained her bachelor degree in Mathematics with honors in 2001, Master in Mathematics in 2002 and $\mathrm{PhD}$ in Mathematics in 2009. Her current research interest includes fuzzy mathematics, topology and medical mathematics. 


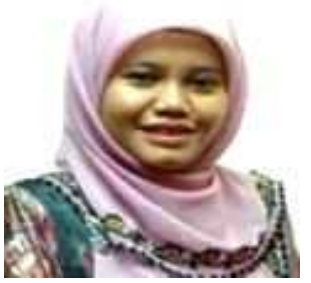
Haslinda $\begin{array}{r}\text { Ibrahim } \\ \text { bachelor }\end{array}$
obtained her in Mathematics
degree
in 1992(Michigan State University, USA), Master in Mathematics in 1996(Indiana State University) and $\mathrm{PhD}$ in Mathematics in 2003 (Southern Illinois University, USA). She is well known for her profound contributions in combinatorics domain especially in Combinatorial Design Theory. Her current research interests include graph theory, combinatorial design theory, enumerative theory and optimization theory and Fuzzy Theory. Her work experiences include deputy dean of postgraduate, head of Mathematics Department, and Coordinator for Postgraduate program. She has been awarded several awards such as University Best Research Award (Universiti Utara Malaysia), Dissertation Research Award (Southern Illinois University, USA), Outstanding PhD Teaching Assistant (Southern Illinois University, USA) and Bronze Medal Award, 9th Malaysia Technology Expo: Invention and Innovation Award (Malaysia). She is also an editorial board for Mathematical Sciences Letter, Journal of Mathematics Research and International Journal of Modern Mathematical Sciences. She has published over 70 articles in journals and conference proceedings. 